\title{
Breast Surgery with Application of Doughnut Mastopexy Lumpectomy Technique
}

\author{
Kyoichi Matsuzaki ${ }^{1,2}$ \\ ${ }^{1}$ Department of Plastic and Reconstructive Surgery, Kawasaki Municipal Tama Hospital, Kawasaki, Japan; ${ }^{2}$ Department of Plastic \\ and Reconstructive Surgery, St. Marianna University School of Medicine, Kawasaki, Japan. \\ Email: k4matsu@marianna-u.ac.jp
}

Received June $5^{\text {th }}$, 2012; revised July $6^{\text {th }}$, 2012; accepted August $3^{\text {rd }}, 2012$

\begin{abstract}
Purpose: Doughnut mastopexy lumpectomy (DML) is a breast resection technique in which a tissue segment is removed and the breast reshaped through a doughnut-shaped de-epithelialized periareolar area. In this study, we attempted to determine whether the DML technique could be useful for other types of breast surgery, in addition to breast cancer lumpectomy. Methods: This study examined a total of 4 patients who underwent the DML technique and were followed up for at least 1 year postoperatively. One patient underwent phyllodes tumor resection, 1 patient underwent removal of a siliconoma, and 2 patients underwent breast reduction mammaplasty. Results: This method enabled en-bloc removal of a large tissue mass or large foreign body that could not be removed through a short periareolar incision. The surgical method of this study enabled the extent of de-epithelialization to be changed according to the size and location of the mass to be excised; good cosmetic results were also obtained. In addition, the surgical method enabled the facile excision of tumors and foreign materials. Conclusions: The DML technique is a useful surgical method that is applicable to other breast surgeries, in addition to breast cancer surgery.
\end{abstract}

Keywords: Breast; Doughnut Mastopexy; Lumpectomy

\section{Introduction}

Scar revision after breast surgery is in greater demand from patients than ever before. Many female patients find it difficult to accept surgery which results in prominent scarring, and this scarring can lead to patient dissatisfaction with the surgical results [1]. Asians in particular are more susceptible to hypertrophic scarring and keloid formation, compared with Caucasians [2,3], and the selection of surgical approach is especially important in this population.

The periareolar incision was first reported by Dufourmentel in 1928. This incision results in less prominent scarring and is used not only for breast augmentation and gynecomastia procedures, but also for benign tumor excision [4]. As a periareolar incision is small, it is difficult to remove a large tissue mass en bloc. Even if such a mass can be removed, there will likely be redundant skin after resection, resulting in an aesthetically poor appearance. Treatment using a periareolar incision is also difficult in cases with the affected sites distant from the areola.

Doughnut mastopexy lumpectomy (DML) is a surgical method which begins with a periareolar incision, but then proceeds to de-epithelialize the periareolar skin in a doughnut shape. An incision is made along a portion of the outer border of the doughnut, and lumpectomy is performed. Remnant tissue is reshaped after lumpectomy to adjust the shape of the breast. Any redundant skin remaining after lumpectomy is adjusted by plication of the outer and inner circumferences of the doughnut-shaped de-epithelialized area [5]. DML is considered a useful alternative to standard lumpectomy for 2 reasons. First, a periareolar scar is more discreet than a scar resulting from direct incision over the affected area, and has an overall more pleasing aesthetic result. Second, glandular volume is rearranged with DML to create a pleasing breast contour, and breast skin is reshaped as well [5]. This study examined whether the DML technique could be useful in breast surgeries other than lumpectomies performed for breast cancer.

\section{Patients}

The subjects were 4 surgical patients who were followed up for at least 1 year postoperatively. One patient was a 50-year-old female who underwent phyllodes tumor resection. Another was a 75-year-old female who underwent removal of silicone, injected 30 years ago for breast augmentation. The other 2 patients were 25- and 39-year- 
old females who underwent breast reduction mammaplasty. All surgeries took place between October 2000 and August 2009.

\section{Surgical Techniques}

To address the issue of excessive skin after resection, the size of the doughnut-shaped epidermal incision was designed preoperatively with the patient in a standing position.

\subsection{Cases with Resection near the Areola}

If the unaffected breast was normal, the cranial aspect of the doughnut's outer circumference was established so that the distance between the sternal notch and the new nipple on the affected breast would be the same as the distance between the notch and the unaffected nipple. If the affected site was near the areola, the doughnut was made in concentric circles and the skin of the breast was stretched from the areola. The skin incision was shallow at the outer and inner circumferences of the doughnut. Subsequently, the epidermis was shaved, using a roundededge scalpel, and removed. The subdermal vascular plexus was preserved to the greatest extent possible. The length of the dermal incision at the outer edge of the doughnut was kept as short as possible, while still allowing for excision of the mass.

\section{Case Presentation}

A 50-year-old female presented with a phyllodes tumor in the right breast (Figure 1(a)). The distance from the sternal notch to the nipple was $1.5 \mathrm{~cm}$ longer in the affected breast, compared with the unaffected breast. Thus, a circle was drawn $1.5 \mathrm{~cm}$ from the areolar margin on the affected breast (Figure 1(b)), and the skin between this circle and the areolar margin was de-epithelialized in a doughnut shape (Figure 1(c)). A semicircular incision was made in the caudal aspect of the outer circumference of the doughnut. The tumor was excised en bloc with normal breast tissue (Figure 1(d)). The remnant breast tissue was reshaped to prevent a concave deformity, and the wound was closed with purse-string suturing of the areolar margin (Figure 1(e)).

\subsection{Cases with Resection Distant from the Areola}

\section{Case Presentation}

A 75-year-old female requested the removal of silicone that had been injected into the right breast for augmentation 30 years prior. The patient did not request silicone removal on the left side due to the presence of a pacemaker. The siliconoma was located mainly cranial to the areola (Figure 2(a)). Thus, periareolar de-epithelialization was performed in a more extensive area at the cranial aspect of the doughnut, and a semicircular incision was made in the cranial aspect of the doughnut's outer circumference. Since the siliconoma invaded the pectoralis major muscle, the surrounding tissue was also excised en bloc to include the muscle and prevent spillage of the gel-like silicone. The size of the excised specimen was $12 \times 12 \times 9 \mathrm{~cm}$ (Figure 2(b)).

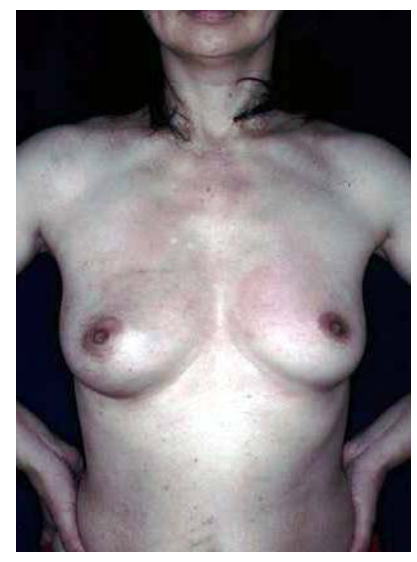

(a)

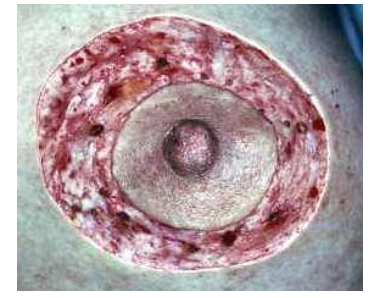

(c)

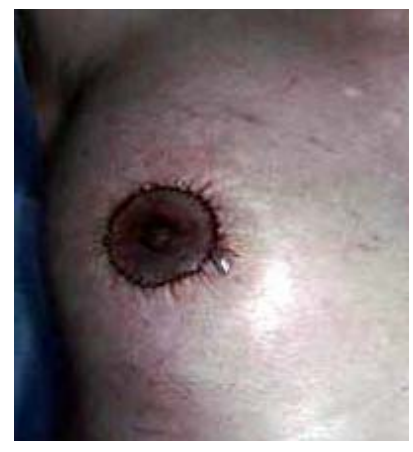

(e)

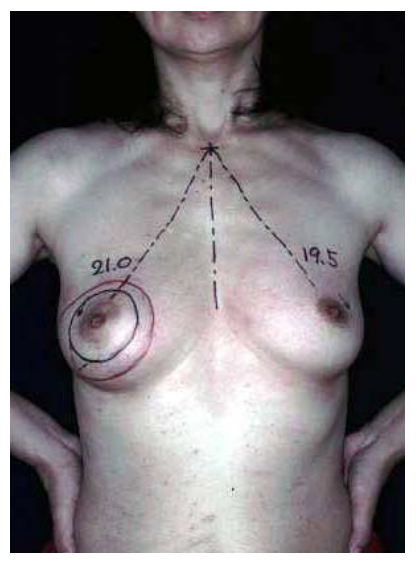

(b)

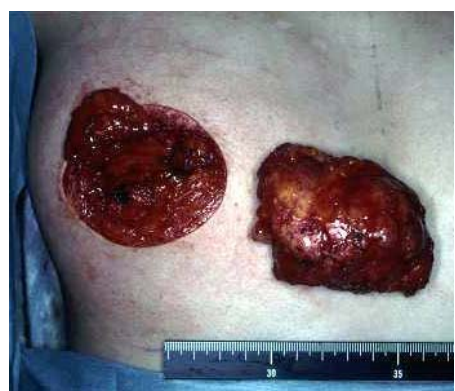

(d)

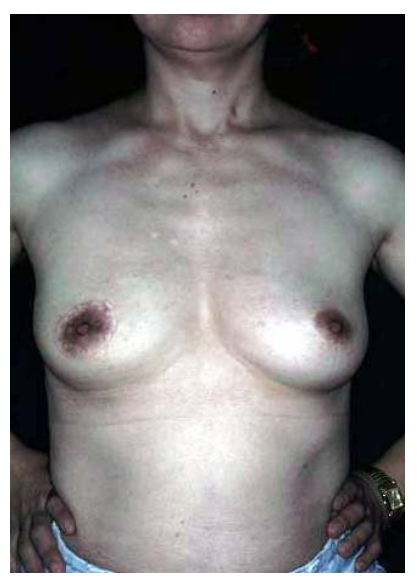

(f)
Figure 1. (a) A 50-year-old female with a phyllodes tumor in the right breast; (b) Preoperative marking. The black circle was drawn $1.5 \mathrm{~cm}$ from the areolar margin. The red circle was the area in contact with the tumor; (c) The skin was de-epithelialized in a doughnut shape; (d) The tumor was excised en bloc with normal breast tissue; (e) The immediate postoperative result; (f) One year postoperatively. 


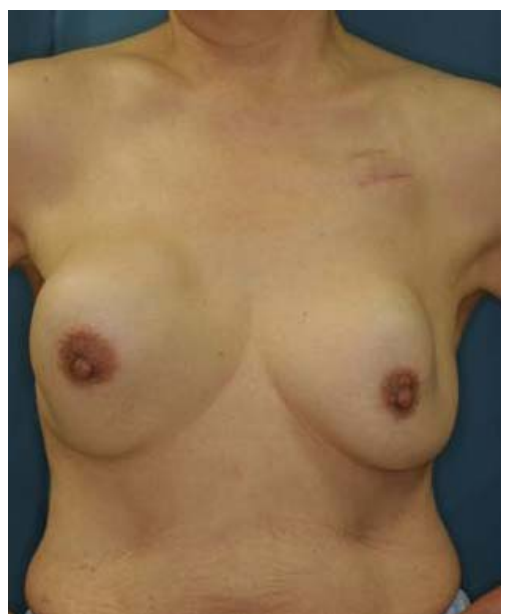

(a)

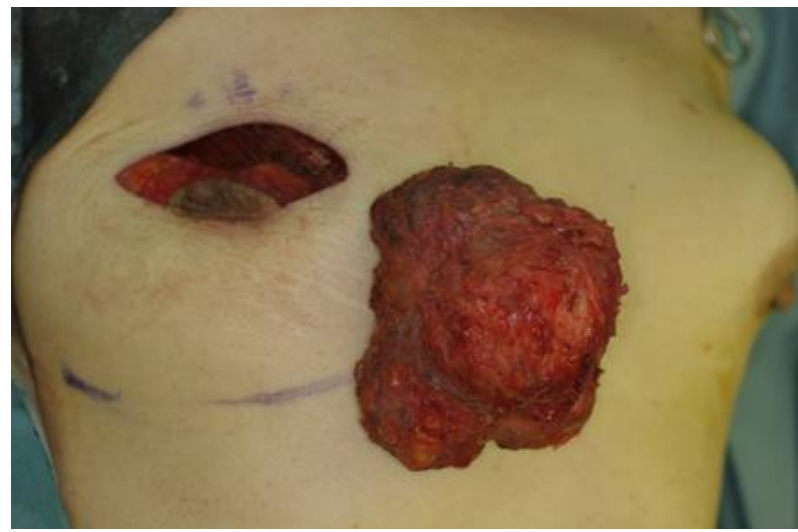

(b)

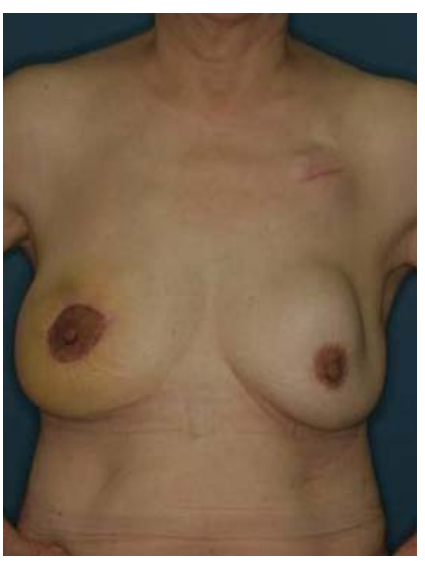

(c)

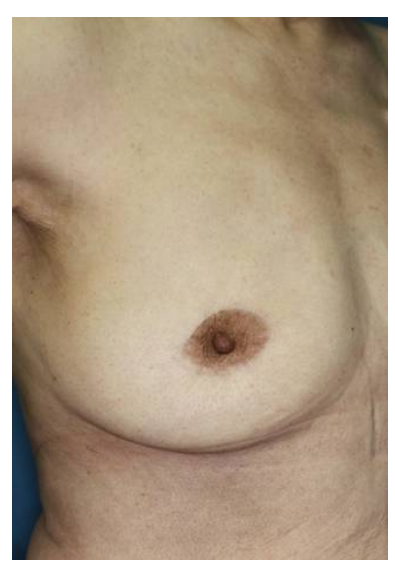

(d)
Figure 2. (a) A 75-year-old female with silicone injected for breast augmentation 30 years prior; (b) The siliconoma with surrounding tissue in the right breast was excised en bloc, including the pectoralis major muscle; (c) Two weeks postoperatively. A gathering was observed at the areolar margin. The patient did not request silicone removal on the left side due to the presence of a pacemaker; (d) Two and a half years postoperatively. The periareolar scar was not conspicuous. In this patient, tension on the skin was not strong at the time of suturing. Thus, there was no enlargement of the areola.

\subsection{Breast Reduction Mammaplasty}

In 1 case of bilateral breast reduction mammaplasty, the distance from the sternal notch to the new location of the areola was determined to be $20 \mathrm{~cm}$. In a second case, breast reduction was performed after post-mastectomy reconstruction of the contralateral breast, which had been affected by breast cancer. In both cases, the desired, symmetric position of the areola was established. The shape of the doughnut was determined based on the amount of breast resection to be performed in its cranial and caudal aspects. During surgery, a dermal incision was made around the entire outer circumference of the doughnut. The breast tissue was resected in the cranial and caudal aspects, according to the Góes method [1]. After the target tissue was excised, the remnant breast tissue was dissected to the minimal extent necessary for reshaping, and suturing was performed. A purse-string closure was used for the outer and inner circumferences of the doughnut. In the 2 cases of breast reduction mammaplasty, areola enlargement was anticipated. A dermal incision was made around the entire outer circumference of the doughnut, increasing potential skin tension on the incision compared with the 2 cases without this circumferential incision. Therefore, a diametrical transareolar U suture using a straight needle was performed at 2 sites where there could be minimum tension on the skin before performing the purse-string closure [6].

\section{Case Presentation}

A 39-year-old female had undergone mastectomy for left breast cancer. The patient requested reconstruction of the left breast and reduction surgery of the right breast (Figure 3(a)). Left breast reconstruction was performed using a superdrainaged transverse rectus abdominis myocutaneous flap. Six months later, the plan was to create a nipple and areola in the reconstructed breast, $31 \mathrm{~cm}$ from the sternal notch. The extent of de-epithelialization for the right breast was marked at a position $3 \mathrm{~cm}$ cranial to the areolar margin. The plan was to resect more breast tissue at the cranial aspect to enhance the effecttiveness of the mastopexy, and so markings were made $2 \mathrm{~cm}$ medial, lateral, and caudal to the areolar margin and an ovoid was drawn connecting the four points (Figure 3(b)). The skin was de-epithelialized in the doughnut area (Figure $\mathbf{3 ( c )}$ ). An incision was made along the doughnut's outer circumference, and the breast resection was performed (Figure 3(d)). Enlargement of the areolar margin was expected, due to strong skin tension at the suture site. To mitigate this problem, a diametrical transareolar U suture was performed with a straight needle to bring together the dermis of the doughnut's outer circumference at opposite points of the circle, incorporating the subareolar dermis. A second diametrical transareolar 


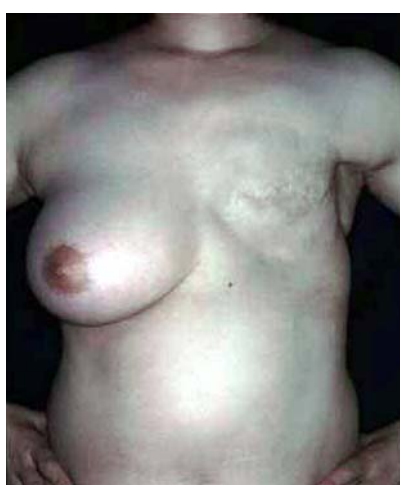

(a)

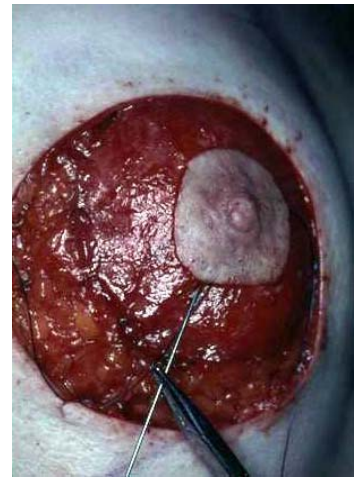

(e)

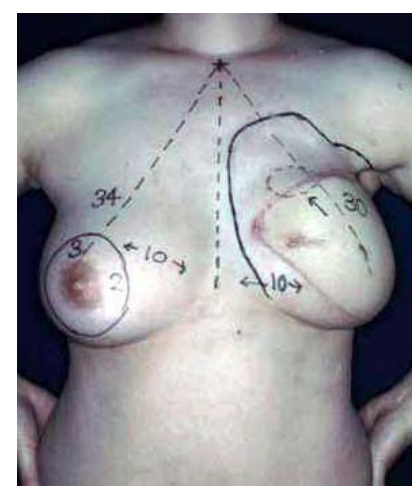

(b)

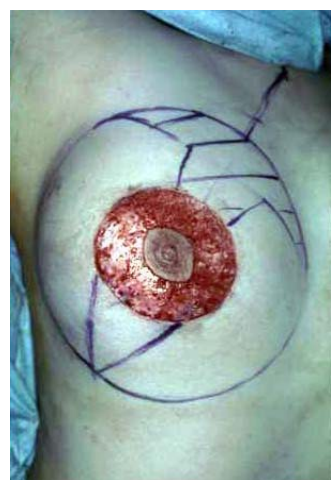

(c)

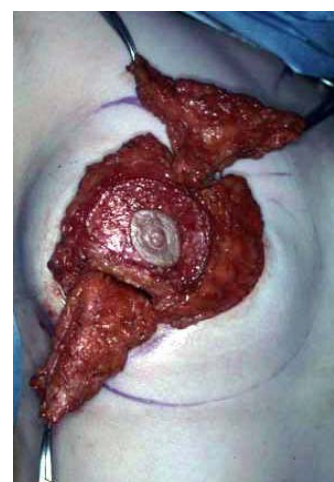

(d)

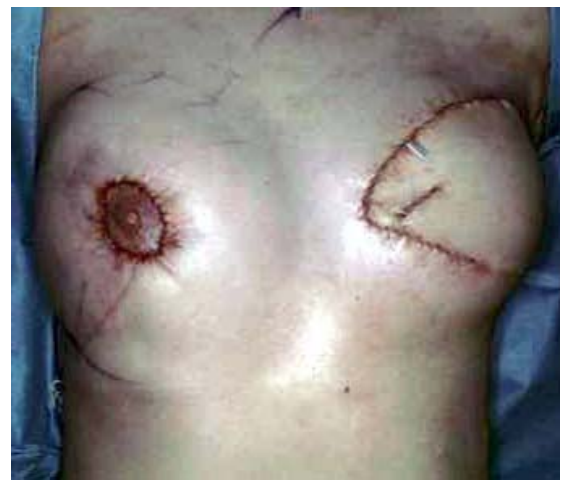

(f)

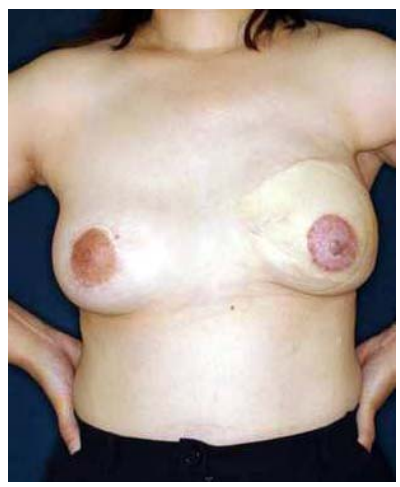

(g)

Figure 3. (a) A 39-year-old female after mastectomy for left breast cancer. The patient requested reconstruction of the left breast and reduction of the right breast; (b) Six months after left breast reconstruction. Preoperative marking for breast reduction mammaplasty; (c) The skin was de-epithelizalized in the doughnut area. The cranial and caudal aspects of the planned extent of breast resection were marked; (d) An incision was made along the doughnut's outer circumference, and breast tissue resection was performed; (e) A diametrical transareolar U suture was performed with a straight needle; (f) The immediate postoperative result. A hypertrophic scar of the left reconstructed breast was resected, and suturing was performed; (g) Two and a half years postoperatively. During this period, a skate flap was used to create a nipple in the left reconstructed breast, and medical tattooing was performed for the nipple and areola.

U suture was placed along a line perpendicular to the first (Figure 3(e)).

\section{Results}

The surgical technique in this study enabled en-bloc removal of a large tissue mass or foreign material through the doughnut's outer circumference incision. Such large specimens cannot be removed en bloc using a small periareolar incision.

Soon after surgery in all patients, there was significant gathering in the sutured area due to the difference between the doughnut's inner and outer circumferences. Six months to 1 year postoperatively, the gathering was no longer conspicuous (Figures 2(c) and (d)).

In long-term follow-up, the areola enlarged in all patients except the patient who had undergone siliconoma removal (Figure 2(d)). No patients had any scarring other than along the areolar margin; these scars were not conspicuous. The patients were satisfied with the shape of their breasts, and there were no sensory disturbances in the nipple or areola (Figures 1(f), 2(d) and 3(g)).

\section{Discussion}

A recent report compared the excision of fibroadenomas of $3 \mathrm{~cm}$ or smaller using a periareolar incision or an incision over the tumor. This report described 76 patients who underwent excision through a periareolar incision and 82 patients who had an incision directly over the tumor [4]. At an early stage, the disadvantages in the periareolar incision group were a longer operating time by 2 minutes, more blood loss by $10 \mathrm{~mL}$, and more severe skin flap bruising. The periareolar incision group had more patients with a disturbance of nipple sensation; this disturbance was seen more frequently when a lateral incision was used. Nipples and their surrounding areolas are innervated by the lateral and anterior cutaneous branches of the third, fourth, and fifth intercostal nerves. Therefore, special precautions are required when making a lateral incision [4,7]. Mammary duct damage can be avoided by careful dissection of the area between the 
subcutaneous fat and breast tissue, in the approach to the tumor. Even with all the aforementioned disadvantages, periareolar incisions have the advantage of a better cosmetic result after removal of fibroadenomas of $3 \mathrm{~cm}$ or smaller [4].

Since the length of a periareolar incision is limited, it is not suitable for a large mass requiring excision. DML has been reported to yield good cosmetic results after tumor excision and excision of normal breast tissue, just as it does in breast cancer surgery. In this study, tumors and foreign materials were excised using the DML technique. If the mass was near the areola, the doughnut incisions were made in concentric circles. If the mass to be excised was far from the areola, concentric circles would stretch and deform the skin on the affected side; in such cases, the doughnut area on the affected side was enlarged, and the resected amount of skin was adjusted. With the DML technique, the outer circumferential incision can be made much longer, facilitating tumor resection. The advantage of this method is that the incision line can be changed according to the size and location of the matter to be excised.

Unlike surgery for benign tumors, in surgery for breast cancer a large amount of normal breast tissue attached to the tumor is excised in order to prevent recurrence. This leaves excess skin behind. Even with benign tumors, the skin can become stretched due to tumor enlargement; similarly, the skin can become stretched if a foreign body is present for a long duration. Because of this excess skin retained after resection, a satisfactory cosmetic result cannot be obtained by excision of a subcutaneous tumor or foreign material alone. The DML technique solves that problem with the adjustable nature of the doughnut skin resection.

Breast reduction mammaplasty and mastopexy using periareolar techniques are surgical methods based on the same concept as the DML technique used in this study. Typical surgical methods are the periareolar Benelli mastopexy [8] and the Góes periareolar technique [1]. In the periareolar Benelli mastopexy, a dermal incision is made along the doughnut's outer circumference, where the epidermis has been resected, from the 2 o'clock to the 10 o'clock position. The skin is dissected from this area down to the inframammary fold. The central and caudal breast tissues are usually the areas resected, then the sagging breast is elevated cranially and fixed to the chest wall. In the Góes periareolar technique, a dermal incision is made around the entire outer circumference of the doughnut, and subsequently the skin of the breast is dissected and elevated from the breast tissue. The cranial and caudal breast tissues are resected and the remnant tissue is reshaped. The remnant doughnut-shaped dermis in the periareolar region is dissected from the breast tissue to create a dermal flap; this flap is then stretched over the surrounding tissue to cover the breast mound as extensively as possible. The caudal aspect of the dermal flap is fixed to the pectoralis major muscle and the cranial aspect is fixed to the connective ligaments to enhance the effectiveness of the mastopexy. The aforementioned steps are the original method described by Góes [1], however, the use of a dermal flap alone results in laxity due to insufficient fixation of the breast mound. Thus, Góes subsequently reported a modified method in which mesh was used to cover the entire breast mound, over the dermal flap, for fixation [9]. The method used in our present report differs from the original method of Góes in the following ways. In our series, the 2 cases of breast reduction mammaplasty did not have sufficiently severe breast enlargement or sagging to require a mesh or dermal flap. In addition, it was unnecessary to dissect a doughnut-shaped dermal flap from the breast tissue and thus interrupt the blood flow to the nipple and areola.

In our study, doughnut mastopexy was performed in 4 breast surgeries that did not involve lumpectomy. Good results were obtained in all 4 cases. Further studies will be conducted in cases with different disease entities and in greater numbers of cases to continue to examine the usefulness of this method.

\section{REFERENCES}

[1] J. C. Góes, "Periareolar Mammoplasty: Double-Skin Technique,” Breast Disease, Vol. 4, No. 2, 1991, pp. 111-127.

[2] H. P. Dustan, "Does Keloid Pathogenesis Hold the Key to Understanding Black/White Differences in Hypertension Severity?” Hypertension, Vol. 26, No. 6, 1995, pp. 858862.

[3] D. D. Datubo-Brown, "Keloids: A Review of the Literature,” British Journal of Plastic Surgery, Vol. 43, No. 1, 1990, pp. 70-77. doi:10.1016/0007-1226(90)90047-4

[4] X. F. Liu, J. X. Zhang, Q. Zhou, F. Chen, Z. M. Shao and C. Lu, "A Clinical Study on the Resection of Breast Fibroadenoma Using Two Types of Incision,” Scandinavian Journal of Surgery, Vol. 100, No. 3, 2011, pp. 147-152.

[5] P. L. Giacalone, O. Dubon, P. Roger, N. El Gareh, S. Rihaoui and J. P. Daurés, "Doughnut Mastopexy Lumpectomy versus Standard Lumpectomy in Breast Cancer Surgery: A Prospective Study,” European Journal Surgical Oncology, Vol. 33, No. 3, 2007, pp. 301-306. doi:10.1016/j.ejso.2006.09.014

[6] J. C. Grotting, A. P. Marx and S. M. Chen, "Mastopexy," In: S. J. Mathes, Ed., Plastic Surgery, Saunders Elsevier, Philadelphia, 2006, pp. 47-86.

[7] I. Schlenz, R. Kuzbari, H. Gruber and J. Holle, “The Sensitivity of the Nipple-Areola Complex: An Anatomic Study," Plastic and Reconstructive Surgery, Vol. 105, No. 3, 2000, pp. 905-909. doi:10.1097/00006534-200003000-00012

[8] L. Benelli, “A New Periareolar Mammaplasty: The 'Round Block’ Technique,” Aesthetic Plastic Surgery, Vol. 14, 
No. 2, 1990, pp. 93-100. doi:10.1007/BF01578332

[9] J. C. Góes, "Periareolar Mammaplasty: Double Skin Technique with Application of Polyglactine or Mixed Mesh,"
Plastic and Reconstructive Surgery, Vol. 97, No. 5, 1996, pp. 959-968. 